\title{
Étude des lacunes dans la surveillance d'une petite éclosion de rougeole à Toronto, Canada
}

\author{
Gournis $E^{1,2^{*}}$, Shane $A^{1,3}$, Shane $E^{1}$, Arthur $A^{1}$, Berger $L^{1,2}$
}

\section{Résumé}

Début 2015, une éclosion de dix cas confirmés de rougeole s'est produite à Toronto, en Ontario. Dans le cadre de l'intervention contre l'éclosion, le personnel du Bureau de santé publique de Toronto a mené des activités traditionnelles et complémentaires d'enquête sur les cas. Malgré ces efforts considérables, et contrairement à de nombreuses éclosions précédentes de rougeole au Canada, ni le cas source ni aucun lien épidémiologique confirmé entre les cas n'ont pu être identifiés. L'enquête sur l'éclosion a mis en lumière des lacunes potentielles dans la surveillance actuelle de la rougeole et des suggestions d'approches à adopter pour les enquêtes ultérieures : utilisation systématique des médias sociaux et d'autres ressources horodatées pour approfondir l'enquête sur les cas, communication ciblée précoce et répétée avec les partenaires de soins primaires pour améliorer la détection des cas, et efforts continus pour accroitre et maintenir une couverture vaccinale suffisante pour interrompre la transmission de la maladie.

\author{
Affiliations \\ ${ }^{1}$ Contrôle des maladies \\ transmissibles, Bureau de santé \\ publique de Toronto, Toronto \\ (Ontario) \\ 2 École de santé publique Dalla \\ Lana, Université de Toronto, \\ Toronto (Ontario) \\ ${ }^{3}$ Programme canadien \\ d'épidémiologie sur le terrain, \\ Agence de la santé publique du \\ Canada, Ottawa (Ontario)
}

*Correspondance : egourni@ toronto.ca

Citation proposée : Gournis E, Shane A, Shane E, Arthur A, Berger L. Étude des lacunes dans la surveillance d'une petite éclosion de rougeole à Toronto, Canada. Relevé des maladies transmissibles au Canada

2016;42:163-5. https://doi.org/10.14745/ccdr.v42i07a02f

\section{Introduction}

Le 28 janvier 2015, le Bureau de santé publique de Toronto a été informé d'un cas de rougeole confirmé en laboratoire. Neuf autres cas confirmés ont été signalés peu après. Les données probantes moléculaires étayaient l'hypothèse selon laquelle tous les cas provenaient d'une seule importation, mais cela n'a pas pu être confirmé par les données épidémiologiques.

Le présent article étudie les lacunes dans la détection des cas de rougeole et dans l'investigation épidémiologique qui ont été repérées pendant l'enquête sur cette éclosion. II décrit les activités épidémiologiques complémentaires mises en œuvre pour combler ces lacunes et envisage les répercussions pour les futures activités d'intervention en cas d'éclosion.

\section{L'éclosion}

Une description détaillée de l'épidémiologie de cette éclosion et de l'intervention de santé publique mise en œuvre pour y répondre sera fournie ailleurs (S. Thomas et al. Measles outbreak with unique genotyping). En résumé, 10 cas confirmés de rougeole similaires du point de vue génotypique ont été signalés au Bureau de santé publique de Toronto entre le 28 janvier et le 17 février 2015. Un système de gestion d'incident a été activé pour gérer et coordonner les activités d'intervention contre l'éclosion. Tous les cas et tous les contacts ont fait l'objet d'une enquête et ont été gérés conformément au Protocole concernant les maladies infectieuses de l'Ontario (1). Ces mesures ont compris le suivi de 1548 contacts et la mise en place de 10 cliniques de prophylaxie post-exposition. Aucun cas secondaire n'a été détecté parmi les contacts. Les données de l'enquête systématique sur les cas n'ont pas permis de révéler la source de l'éclosion de rougeole.

\section{Investigations épidémiologiques complémentaires}

Lorsqu'il est apparu que l'on ne trouvait pas de liens épidémiologiques entre les cas, les épidémiologistes du Bureau de santé publique de Toronto ont mené des activités complémentaires pendant l'enquête de santé publique. Ces activités avaient pour but de trouver davantage de renseignements fournis par les cas sur l'exposition possible afin de mieux comprendre et caractériser la transmission de la rougeole au sein de la communauté. Tout d'abord, on a demandé à un sous-ensemble de cas ou à leurs tuteurs d'utiliser les renseignements des médias sociaux ( $p$. ex. publications sur Instagram) et les relevés en ligne de leurs comptes bancaires respectifs pour les aider à se souvenir de leurs activités pendant les périodes possibles d'acquisition et de transmission. Chaque entretien téléphonique supplémentaire a pris deux à trois heures de plus par cas (y compris le temps nécessaire pour valider les adresses). Cela a permis de repérer un certain nombre de lieux intéressants supplémentaires en plus de ceux établis dans les entretiens initiaux. En outre, l'enquête a eu recours à l'outil Pajek de visualisation des réseaux sociaux (2) pour détecter les expositions se chevauchant. Étant donné que la liste des expositions s'est allongée et que l'examen manuel est devenu chronophage et onéreux, l'inclusion de la visualisation des réseaux sociaux dans le cycle de déclaration systématique a abouti à une méthode plus rapide et plus systématique pour repérer les liens épidémiologiques potentiels. 


\section{Lacunes dans la détection}

En dépit des mesures traditionnelles et complémentaires d'enquête sur les cas, les données probantes sont restées insuffisantes pour confirmer la source de l'éclosion ou l'existence de liens épidémiologiques entre les cas. Étant donné que le Laboratoire national de microbiologie (LNM) avait caractérisé les cas comme étant similaires du point de vue génotypique et probablement dus à un seul événement d'importation (Communication personnelle, Alberto Severini, Laboratoire national de microbiologie, 31 mars 2016), il était évident qu'une lacune subsistait dans la détection des cas et dans l'enquête. Bien qu'il ne soit pas rare que le cas source d'une éclosion ne soit pas détecté au Canada, une petite éclosion communautaire contenue de rougeole sans lien épidémiologique identifié constitue un fait inhabituel (3).

Plusieurs hypothèses pourraient expliquer ces constats. II est possible que les personnes atteintes de la rougeole ne se soient jamais présentées dans un établissement du système de soins de santé. Il est également possible qu'elles s'y soient présentées, mais qu'elles n'aient pas été identifiées comme des cas soupçonnés de rougeole et n'aient donc pas subi les analyses appropriées. Dans ces deux scénarios, il se peut que les personnes aient ressenti des symptômes atténués, ou subcliniques, éventuellement dus à l'échec d'un vaccin secondaire (4), et n'aient pas répondu à la définition classique d'un cas clinique ou de laboratoire pour la rougeole. II est aussi possible que les procédés traditionnels et complémentaires d'investigation épidémiologique sur les cas et les outils utilisés dans cette enquête aient été insuffisants pour repérer l'exposition ou les expositions communes, compte tenu de la transmissibilité élevée du virus, de la mobilité de la population et de l'environnement urbain où résidaient les cas. Les entretiens supplémentaires facilités par les médias sociaux n'ont été effectués que sur un sous-ensemble de cas. Si ces méthodes avaient été appliquées aux 10 cas, d'autres liens auraient peut-être été établis.

\section{Discussion}

Malgré les enquêtes systématiques et approfondies menées sur une éclosion de rougeole limitée à 10 cas confirmés en laboratoire à Toronto, en Ontario, ni la source ni aucun lien épidémiologique confirmé n'ont pu être identifiés. Il est important d'envisager les répercussions de ces constats pour la surveillance de la rougeole et les efforts d'élimination de la maladie au Canada.

Afin de documenter suffisamment le statut d'élimination de la rougeole au Canada, il est essentiel de réduire le plus possible le nombre de cas sporadiques ou de source inconnue et de veiller à mener une enquête consciencieuse et à bien comprendre les événements de transmission. Même s'il a été suggéré que les cas subcliniques pourraient être moins infectieux que les cas cliniques, la détection de tous les cas demeure importante afin de comprendre les éclosions et la dynamique de la transmission (5).

Bien que les éclosions de rougeole au Canada soient rares et de petite taille, les ressources nécessaires pour gérer ces éclosions sont considérables, en particulier sur le plan de la gestion du suivi des personnes sous investigation, des cas soupçonnés et des contacts dans un contexte de couverture vaccinale élevée (6). Par exemple, après que l'éclosion a été déclarée, le Bureau de santé publique de Toronto a reçu de nombreux signalements de personnes sous investigation. Ces personnes ne répondaient pas à la définition de cas de l'éclosion, mais il a toutefois fallu engager des ressources de santé publique et de laboratoire non négligeables pour écarter la possibilité de la maladie chez ces personnes. Trouver un équilibre entre, d'une part, le degré acceptable de sensibilité de la surveillance requis pour caractériser et interrompre la transmission et, d'autre part, l'allocation adéquate des ressources nécessaires pour maintenir ce degré de sensibilité constitue un enjeu clé, en particulier dans les zones où la couverture vaccinale est élevée et homogène.

Les lacunes repérées dans le présent résumé laissent à penser que d'importantes occasions existent pour améliorer l'identification des cas et l'investigation épidémiologique sur la rougeole. Les ressources requises pour des activités supplémentaires doivent être comparées avec ce qui est déjà nécessaire pour respecter les normes actuelles d'investigation sur les éclosions de rougeole.

Pour améliorer la capacité à décrire précisément l'épidémiologie de la rougeole chez les cas confirmés, il est nécessaire de continuer à mettre l'accent sur la collecte de renseignements complets sur l'exposition (aussi bien sur l'acquisition que sur la transmission) pendant les entretiens avec les cas. Cela peut inclure de demander systématiquement aux cas de passer en revue des ressources horodatées ( $p$. ex. relevés de compte bancaire et de carte de crédit ou sites de médias sociaux) pour les aider à se souvenir de leurs activités et des lieux fréquentés pendant leurs périodes d'exposition et de transmissibilité, ainsi que d'utiliser des outils de visualisation des réseaux sociaux pour gérer la complexité associée à ces renseignements supplémentaires.

Pour combler le manque potentiel de sensibilité décelé lors de l'enquête sur cette éclosion, une communication précoce, répétée et active avec les réseaux de soins primaires et de services d'urgence pendant les éclosions de rougeole peut contribuer à renforcer la vigilance aux principaux signes et symptômes qui déclenchent les procédures adéquates d'analyse en laboratoire. Cela a été fait pendant l'éclosion de 2015 aux moyens d'alertes et de communications avec les prestataires de soins primaires. Si moins de déclarations de personnes sous enquête sont reçues car les médecins savent quels cas déclarer et faire dépister, les ressources de santé publique pourront être réattribuées à des activités épidémiologiques supplémentaires. Pour améliorer la détection des cas de rougeole en dehors des périodes d'éclosion (c.-à-d. afin de détecter les cas sources), il est important de rappeler aux prestataires de soins de santé que les symptômes peuvent être atténués chez les personnes immunisées auparavant et de prendre en considération les antécédents de voyage en provenance de régions où la rougeole est endémique pour éclairer le diagnostic différentiel.

Des efforts continus visant à accroître la couverture vaccinale et l'accès aux dossiers électroniques confirmant le statut d'immunisation pourront permettre aux organismes de santé publique, d'une part, de compter sur l'immunité collective pour interrompre la transmission de la rougeole après une importation et, d'autre part, de rééquilibrer les ressources de santé publique 
entre la gestion des contacts et l'enquête sur les personnes sous examen ainsi que sur les cas soupçonnés et confirmés.

\section{Conclusion}

En dépit des activités systématiques et complémentaires d'enquête sur les cas, le Bureau de santé publique de Toronto n'a pas réussi à confirmer l'existence de liens épidémiologiques entre les 10 cas de l'éclosion. Les lacunes dans la détection des cas et dans les activités d'enquête que cette éclosion inhabituelle a révélées pourront servir de base pour éclairer les activités d'intervention contre les éclosions à l'avenir.

\section{Remerciements}

Nous souhaitons remercier tout le personnel du Bureau de santé publique de Toronto qui a contribué à l'enquête et à la gestion de cette éclosion, l'équipe de Santé publique Ontario qui a aidé à coordonner l'enquête et l'intervention au niveau provincial, et le personnel du Laboratoire national de microbiologie pour son travail et pour les renseignements sur la caractérisation phylogénique des cas.

\section{Conflit d'intérêts}

Aucun.

\section{Financement}

Aucun.

\section{Références}

1. Ministry of Health and Long-Term Care. Infectious diseases protocol. Appendix A: disease-specific chapters. Chapter: Measles. Toronto (ON): The Ministry [revised 2014 Aug]. http://www.health.gov.on.ca/en/pro/programs/publichealth/ oph_standards/docs/measles_chapter.pdf.

2. Batagelj $\mathrm{V}$, Mrvar A. Pajek - program for large network analysis. 2000. http://vlado.fmf.uni-lj.si/pub/networks/pajek/.

3. Agence de la santé publique du Canada. Lignes directrices pour la prévention et le contrôle des éclosions de rougeole au Canada. Ottawa (ON) : Agence de la santé publique du Canada [Date de modification : le 4 avril 2013]. http://www. phac-aspc.gc.ca/im/vpd-mev/measles-rougeole-mg-fra.php.

4. De Serres G, Boulianne N, Defay F, Brousseau N, Benoît $M$, Lacoursière $S$, et al. Higher risk of measles when the first dose of a 2-dose schedule of measles vaccine is given at 12-14 months versus 15 months of age. Clin Infect Dis 2012;55(3):394-402.

5. Glass K, Grenfell BT. Waning immunity and subclinical measles infections in England. Vaccine 2004;22(29-30):41106.

6. Wilson SE, Fediurek J, Seo CY, Deeks SL, Lim GH. Immunization coverage report for school pupils: 2012-13 school year. Toronto (ON): Public Health Ontario; 2014. https://www.publichealthontario.ca/en/eRepository/ Immunization_coverage_report_2012-13.pdf.

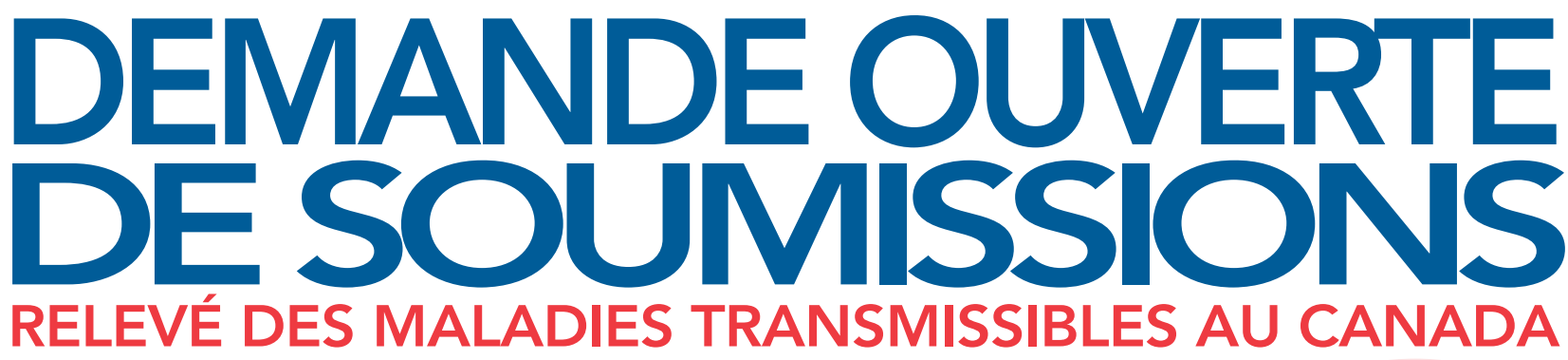

En 2015, nous avons publié des articles d'auteurs provenant de la majorité des provinces et des territoires.

Nous vous invitons à soumettre des articles contenant des renseignements qui font autorité sur les maladies infectieuses, qui éclaireront les politiques, les programmes et les pratiques.

\footnotetext{
Visitez : www.phac-aspc.gc.ca/publicat/ccdr-rmtc/ia-ra-fra.php 